\title{
Correction to: Characterization of 4.2-km-Deep Fractured Granodiorite Cores from Pohang Geothermal Reservoir, Korea
}

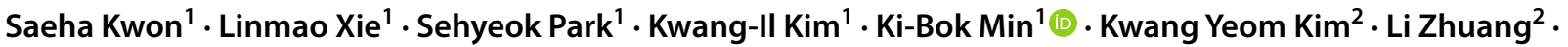 \\ Jaiwon $\mathrm{Choi}^{3} \cdot$ Hanna Kim ${ }^{4} \cdot$ Tae Jong Lee ${ }^{4}$
}

Published online: 31 July 2019

(c) Springer-Verlag GmbH Austria, part of Springer Nature 2019

\section{Correction to: Rock Mech Rock Eng (2019) 52(3):771-782 https://doi.org/10.1007/s00603-018-1639-2}

We note that in the published article the number given in the Acknowledgements is incorrect. It should have been written: "The research was supported by a Grant (No. 20123010110010)."

The original article can be found online at https://doi.org/10.1007/ s00603-018-1639-2.

Ki-Bok Min

kbmin@snu.ac.kr

1 Seoul National University, (08826) 1, Gwanak-ro, Gwanak-gu, Seoul, Republic of Korea

2 Korea Institute of Civil Engineering and Building Technology, (10223) 283, Goyang-daero, Ilsanseo-gu, Goyang, Gyeonggi, Republic of Korea

3 NEXGEO Inc, (05703) 16-15, Garakbon-Dong, Songpa-Gu, Seoul, Republic of Korea

4 Korea Institute of Geoscience and Mineral Resources, (34132) 124, Gwahak-ro, Yuseong, Daejeon, Republic of Korea 